\title{
A simple approach to implementing multiplication with small tables
}

\author{
Biing-Feng Wang, Chuen-Liang Chen and Gen-Huey Chen \\ Department of Computer Science and Information Engineering, National Taiwan University, Taipei, Taiwan, ROC \\ Communicated by K. Ikeda \\ Received 15 October 1990
}

\begin{abstract}
Wang, B.-F., C.-L. Chen and G.H. Chen, A simple approach to implementing multiplication with small tables, Information Processing Letters 37 (1991) 327-329.

A new approach for implementing multiplication with a small table is presented. This approach is much simpler and thus easier to be implemented.
\end{abstract}

Keywords: Data structures, information retrieval

The availability of fast and relatively inexpensive ROM's (read only memories) has made table lookup approach attractive for generating the product of two binary integers [2]. A multiplication table stored in a ROM contains the products of all pairs of the input operands. Using two operands as indices, we can look up the table for their product quickly. The primary difficulty is that the table size can be prohibitively large.

Suppose the multiplicand and multiplier contain $n$ bits each. The multiplication table then contains $2^{2 n}$ entries. For example, the table contains $2^{26}(=65536)$ entries as $n=8$. Because the table size grows quickly as $n$ increases, the table lookup approach is adopted only when $n$ is small. However, Hemel [1] and Ling [3] have suggested approaches to reducing the table size considerably and therefore the table lookup approach can be used cost-effectively for larger $n$. In this paper, a new approach is presented to implement multiplication with a small table. Compared with Hemel's and Ling's approaches, the new one is much simpler and thus easier to be implemented.

\section{Hemel's approach [1]}

Let $A=a_{n-1} a_{n-2} \cdots a_{1} a_{0}$ ( $n$ is even) be the multiplicand and $B=b_{n-1} b_{n-2} \cdots b_{1} b_{0}$ the multiplier. To reduce the table size, Hemel partitioned $A$ (respectively $B$ ) into two suboperands

$A_{1}^{(2)}=a_{n-1} a_{n-2} \cdots a_{n / 2}$

and

$A_{2}^{(2)}=a_{n / 2-1} a_{n / 2-2} \cdots a_{0}$

(respectively

$B_{1}^{(2)}=b_{n-1} b_{n-2} \cdots b_{n / 2}$

and

$\left.B_{2}^{(2)}=b_{n / 2-1} b_{n / 2-2} \cdots b_{0}\right)$.

Then, product $A B$ can be compitted as

$$
\begin{aligned}
A B= & \left(2^{n / 2} A_{1}^{(2)}+A_{2}^{(2)}\right)\left(2^{n / 2} B_{1}^{(2)}+B_{2}^{(2)}\right) \\
= & 2^{n} A_{1}^{(2)} B^{(2)}+2^{n / 2}\left(A_{1}^{(2)} B_{2}^{(2)}+A_{2}^{(2)} B_{1}^{(2)}\right) \\
& +A_{2}^{(2)} B_{2}^{(2)}
\end{aligned}
$$


where three additions, four table lookups (for the values of subproducts $A_{i}^{(2)} B_{j}^{(2)}, i=1,2, j=1,2$ ) and some shift operations (performed by hardware to implement multiplications by $2^{n}$ and $2^{n / 2}$ ) are needed. Because the operands are all of length $n / 2$, the resulting table is of size $2^{n}$.

\section{Ling's approach [3]}

Let $X=\lfloor(A+B) / 2\}, \quad Y=\lfloor|A-B| / 2\}$ and define a function $F$ as

$F(k)=k(k+1) / 2$.

Ling has shown that product $A B$ can be computed as

$A B=2 F(X)-2 F(Y)-\bar{f} g B-\bar{f} \bar{g} A$,

where $\bar{f}(\bar{g})$ is the complement of $f(g), f=0$ if $A+B$ is even and $f=1$ otherwise, and $g=1$ if $A \geqslant B$ and $g=0$ otherwise. If a table of size $2^{n}$ is used to store the function $F$, the cost to generate $A B$ includes four additions, two table lookups and some hardware operations required to perform shift operations and determine $\bar{f}, \bar{g}$.

Ling's approach can be implemented in a pipeline fashion and allows a product to be generated in four submachine cycles. The timing diagram is given in Fig. 1.

\section{A new approach}

Define a function $H$ as follows:

$H(k)=k^{2}$.
Since $(A-B)^{2}=A^{2}+B^{2}-2 A B$, we have

$A B=(H(A)+H(B)-H(A-B)) / 2$.

If a table of size $2^{n}$ is used to store the function $H$, computing $A B$ requires three additions, three table lookups and one shift operation. A pipeline implementation of this approach is shown in Fig. 2. Like Ling's approach, a product can be generated in four submachine cycles.

Besides simplicity and easy implementation (less hardware cost is required), the new approach has the following advantages. First, computing the square of a number is a basic operation in many applications, which now can be performed by looking up the table directly. Second, it is preferable to vector-scalar multiplication, which is frequently executed by computers for many scientific computations. As an example, only $2 m+1$ table lookups, instead of $3 \mathrm{~m}$, are sufficient to the multiplication of a vector $\left(V_{1}, V_{2}, \ldots, V_{m}\right)$ of length $m$ by an integer $S$, i.e.,

$$
\begin{aligned}
& \left(V_{1}, V_{2}, \ldots, V_{m}\right) \times S \\
& \quad=\left(V_{1} \times S, V_{2} \times S, \ldots, V_{m} \times S\right) .
\end{aligned}
$$

\section{Using a table of size smaller than $2^{n}$}

While $n$ is very large, further reduction of the table size is necessary. In the following discussion, approaches using tables of size smaller than $2^{n}$ are introduced. For simplicity, only numbers of additions and table lookups are counted.

As mentioned in [1], Hemel's approach can be extended by partitioning the two operands each into $k, 2 \leqslant k \leqslant n$, suboperands to further reduce

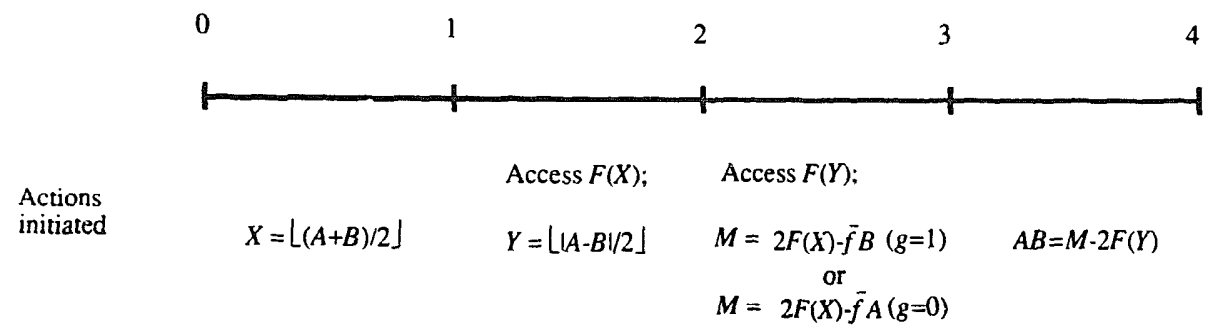

Fig. 1. A timing diagram for Ling's table-assisted multiplication-a pipeline implementation. 


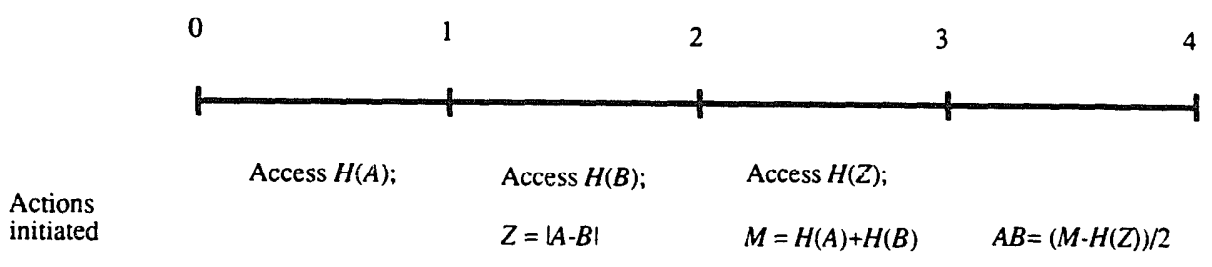

Fig. 2. A timing diagram for the new table-assisted multiplication-a pipeline implementation.

the table size. For example, the table size becomes $2^{n / 2}$ if the operands $A$ and $B$ are partitioned into four suboperands $A_{i}^{(4)}$ 's and $B_{i}^{(4)}$ 's, $i=1, \ldots, 4$. In this example, fifteen additions and sixteen table lookups are necessary to the generation of product $A B$. In general, if the two operands are partitioned into $k$ suboperands each, $2 \leqslant k \leqslant n$, the table size becomes $2^{2 n / k}$ and $k^{2}-1$ additions and $k^{2}$ table lookups are required to generate a product.

A hybrid approach that combines the proposed approach and Hemel's approach can reduce the number of table lookups to $k^{2} / 4+k$ when the table size is $2^{2 n, k}$, where $k$ is even and $2 \leqslant k \leqslant n$. First, the two operands are partitioned into $k / 2$ suboperands each. Then, the $k^{2} / 4$ subproducts are computed by the proposed approach. For example, suppose a table of size $2^{n / 2}$ (i.e., $k=4$ ) is used and the product $A B$ is to be generated. The operands $A$ and $B$ are partitioned into two suboperands $A_{1}^{(2)}, A_{2}^{(2)}$ and $B_{1}^{(2)}, B_{2}^{(2)}$ respectively and then the four subproducts $A_{1}^{(2)} B_{1}^{(2)}, A_{1}^{(2)} B_{2}^{(2)}$, $A_{2}^{(2)} B_{1}^{(2)}$ and $A_{2}^{(2)} B_{2}^{(2)}$ are computed as follows:

$$
\begin{aligned}
& A_{1}^{(2)} B_{1}^{(2)} \\
& \quad=\left(H\left(A_{1}^{(2)}\right)+H\left(B_{1}^{(2)}\right)-H\left(A_{1}^{(2)}-B_{1}^{(2)}\right)\right) / 2,
\end{aligned}
$$

$A_{1}^{(2)} B_{2}^{(2)}$

$$
=\left(H\left(A_{1}^{(2)}\right)+H\left(B_{2}^{(2)}\right)-H\left(A_{1}^{(2)}-B_{2}^{(2)}\right)\right) / 2,
$$

$A_{2}^{(2)} B_{1}^{(2)}$

$$
=\left(H\left(A_{2}^{(2)}\right)+H\left(B_{1}^{(2)}\right)-H\left(A_{2}^{(2)}-B_{1}^{(2)}\right)\right) / 2 .
$$

$A_{2}^{(2)} B_{2}^{(2)}$

$$
=\left(H\left(A_{2}^{(2)}\right)+H\left(B_{2}^{(2)}\right)-H\left(A_{2}^{(2)}-B_{2}^{(2)}\right)\right) / 2 .
$$

Then, the product $A B$ is computed as (1). In this example, fifteen additions and eight table lookups $\left(H\left(A_{1}^{(2)}\right), H\left(A_{2}^{(2)}\right), H\left(B_{1}^{(2)}\right), H\left(B_{2}^{(2)}\right)\right.$ and $H\left(A_{i}^{(2)}\right.$ $\left.\left.-B_{j}^{(2)}\right), i=1,2, j=1,2\right)$ are required. In general, the hybrid approach requires $k^{2}-1$ additions and $k^{2} / 4+k$ table lookups to generate a product when a table of size $2^{2 n / k}$ is used. The hybrid approach requires fewer table lookups than Hemel's approach.

If Ling's approach is used to compute the subproducts instead, then $5 k^{2} / 4-1$ additions and $k^{2} / 2$ table lookups are required to generate a product when a table of size $2^{2 n / k}$ is used. The resulting hybrid approach requires more additions and more table lookups (as $k>4$ ) than the proposed hybrid approach.

\section{References}

[1] A. Hemel, Making small ROMs do math quickly, cheaply and easily, in: W.B. Riley, ed., Electronic Computer Memory Technology (McGraw-Hill, New York, 1971) 133-140.

[2] K. Hwang, Computer Arithmetic (Wiley, New York) 201206.

[3] H. Ling, An approach to implementing multiplication with small tables, IEEE Trans. Comput. 39 (5) (1990) 717-718. 